\title{
On the Enumeration of Majority Games
}

\author{
By John R. Isbell
}

1. Introduction. This paper presents a combinatorial method for the enumeration of all strong weighted majority games, and an arithmetical method for the enumeration of the games having "homogeneous" weights in the sense of von Neumann and Morgenstern [1]. In contrast with the combinatorial method of von Neumann and Morgenstern [1], both of these methods (a) do not generate extra isomorphic copies of the same game, but (b) do generate some unwanted objects (so that tests are required), and (c) are recursive on the number of players. The known (von Neumann and Morgenstern [1], Gurk and Isbell [2]) complete list of 30 strong simple games on $n \leqq 6$ players ( 21 of which are majority games) is supplemented by a complete list of the 114 strong majority games with 7 non-dummy players.

A strong simple game (for the rest of this paper, a game) on a finite set $N$ of players is (1) intuitively, a scheme for distributing power to coalitions of players, all-or-none (this is the sense of "simple"), and so that if the players are partitioned into two parties, one must win (this is the sense of "strong"). (2) Precisely, it is a set of subsets of $N$ called winning sets, such that (a) any set containing a winning set is winning, and (b) for any $S \subset N$, either $S$ or $N-S$ is winning but not both. (3) In this paper, we shall identify two games if there is a one-to-one correspondence between their players identifying their families of winning sets; to name a more definite object one must say, not "the game $G$ ", but "the game $G$ with ordered players $P_{1}, \cdots, P_{n}$ ", or a similar phrase. Beyond this, note that an $n$-player game (e.g. the 435-player game of the House of Representatives) may be converted into an $(n+k)$-player game by adjoining $k$ "voteless" players or dummies. The phrase "an $n$-player game $G$ " does not here imply that all $n$ players are non-dummies. (In the first part of the argument we need games with dummies; afterward we shall exclude them.)

A majority game is a game for which one can assign numerical weights $w_{1}, \cdots$, $w_{n}$ to the players so that the winning sets are precisely those sets which have more than half the total weight. Some of the $w_{i}$ may be negative or zero; it is easy to see that the corresponding players must be dummies. (Since every superset of a winning set wins, a player can have negative weight $w_{i}$ only if $\left|w_{i}\right|$ is so small that it makes no difference.) Given a game $G$, the question whether $G$ is a majority game is effectively decidable, since it turns on a finite system of linear inequalities. No better method than the obvious ones is known. It is clear that every majority game has non-negative integral weights - even positive integral weights, for any weights may be assigned to the dummies provided the weights of the other players are made large enough.

The method described below for enumerating all majority games depends on the determination of the game with ordered players with weights $\left(w_{0}, w_{1}, \cdots, w_{n}\right)$ by the systems $\left(w_{0}+w_{1}, w_{2}, \cdots, w_{n}\right)$ and $\left(w_{0}-w_{1}, w_{2}, \cdots, w_{n}\right)$. The choice

Received 27 June 1957. Supported in part by the Office of Naval Research under Contract N7onr41904 at George Washington University, and in part by the National Science Foundation fellowship. Presented to the Econometric Society, August 1956. 
of weights and orderings gives no trouble. However, the method does not generate weights, but only a combinatorial description of the game. There is an obvious formula for weights for the large game in terms of "suitable" weights for the small games, but we have no version of the method which does not involve both the solution of systems of linear inequalities and the examination of systems which turn out to be inconsistent. There is, however, a fairly simple combinatorial criterion which screens out all the inconsistent systems through $n=7$.

Weights $\left(w_{1}, \cdots, w_{n}\right)$ for a game are called efficient if every winning set $S$ contains a subset which wins by exactly one vote. (This is equivalent to saying that every minimal winning set wins by one vote. Von Neumann and Morgenstern [1] call these "homogeneous" weights, but this term seems likely to lead to confusion.) It is known [3] that efficient weights $w_{i}$ for a game with ordered players are unique; they are non-negative integers; for any other non-negative integral weights $v_{i}$ belonging to the same game with ordered players, $w_{i} \leqq v_{i}$ for all $i$; and when the nonzero weights $w_{i}$ are arranged in non-decreasing order, they grow no faster than the Fibonacci numbers. We call a non-decreasing sequence of positive integers $\left(w_{1}\right.$, $\cdots, w_{k}$ ) an efficient sequence if it is an initial segment of some non-decreasing efficient game weights $\left(w_{1}, \cdots, w_{k}, \cdots, w_{n}\right)$. We find a characterization of these and a recursive arithmetical method for generating them, which generates nothing but efficient sequences. It is necessary, of course, to select those which are actually game weights; for $n=7$ there are 23 games in the 99 efficient sequences. The test for this is simple, and the whole computation is far easier than the other one described above.

It is known [3] that for $n \geqq 4$ there are at least $2^{n-4}$ efficient weighted majority games with $n$ non-dummy players. We obtain the upper bound $(n-1)$ !-actually an upper bound for the number of efficient sequences. There is even more room between the known bounds for general majority games with $n$ non-dummy players; we find there are more than $2^{n}$ for $n \geqq 8$, but no upper bound is known short of $2^{2^{n}}$ (the number of sets of sets of players).

A little experimentation with the methods presented here will quickly show the desirability of an arithmetical method for enumerating all majority games. This would presumably involve a unique choice of weights for each game. For $n \leqq 7$ each $n$-player majority game has minimum non-negative integral weights $w_{i}$. (That is, for any non-negative integral $v_{i}$ defining the same game and order, $w_{i} \leqq v_{i}$ for each $i$.) Such weights are clearly unique. However, they do not always exist, as we show with a certain 12-player game.

To put the classes of games in perspective, consider the author's manuscript list of 559 non-majority 7-player games without dummies, thought to be free from duplications. It was computed by the von Neumann-Morgenstern method some years ago and 109 of the 114 majority games were found.

2. The operators $H_{1}$ and $H_{2}$. We wish to formalize the construction described by the symbol $\left(w_{0}-w_{1}, w_{2}, \cdots, w_{n}\right)$. A motion of a game is a permutation of its players taking winning sets to winning sets. Under the group of motions of the game the players sweep through transitivity sets which we call roles. A court is a majority game with a distinguished role. A natural ordering of the players of a majority game is an ordering $\left(P_{1}, \cdots, P_{n}\right)$ such that the game has weights $w_{i}$ such that $w_{1} \geqq$ 
$w_{2} \geqq \cdots \geqq w_{n}$. However, a natural ordering of the players of a court is an ordering $\left(P_{1}, \cdots, P_{n}\right)$ such that $P_{1}$ is an element of the distinguished role and for some weights $w_{i}, w_{2} \geqq \cdots \geqq w_{n}$.

LemMA 1. If $\left(w_{i}, \cdots, w_{n}\right)$ and $\left(v_{1}, \cdots, v_{n}\right)$ are two systems of weights for a majority game $G$ with ordered players $P_{1}, \cdots, P_{n}$, and $w_{1} \geqq \cdots \geqq w_{n}$, and $\alpha$ is a permutation of the integers from 1 to $n$ such that $v_{\alpha(1)} \geqq \cdots \geqq v_{\alpha(n)}$, then $P_{i} \rightarrow P_{\alpha(i)}$ is a motion of $G$.

Proof. If $w_{i} \geqq w_{j}$ and $S$ is a winning set containing $j$ but not $i$ then replacing $j$ by $i$ in $S$ yields another winning set, since it does not decrease the weight. If also $v_{j} \geqq v_{i}$ then $i$ and $j$ may be exchanged (by a motion leaving all other players fixed). Then the lemma follows by induction.

For any majority game $G$ of $n+1 \geqq 2$ players, we define an $n$-player court $H_{1}(G)$ and an $n$-player game $H_{2}(G)$ as follows. It suffices to define games with ordered players, $H$ with players $Q_{1}, \cdots, Q_{n}, H^{\prime}$ with players $R_{1}, \cdots, R_{n}$, specifying that $H_{1}(G)$ consists of the game $H$ with the role of $Q_{1}$ distinguished and $H_{2}(G)$ is the game $H^{\prime}$. Let $\left(w_{0}, \cdots, w_{n}\right)$ be any system of weights for $G$, arranged so that $w_{0} \geqq \cdots \geqq w_{n}$. We define $H$ by its weights $\left(w_{0}-w_{1}, w_{2}, \cdots, w_{n}\right)$, and $H^{\prime}$ by its weights $\left(w_{0}+w_{1}, \cdots, w_{n}\right)$.

Lemma 2. $H_{1}(G)$ and $H_{2}(G)$ are determined by the game $G$; and together they determine $G$.

Proof. The first half is a routine analysis of definitions, and we omit it. For the converse, let $G$ and $G^{\prime}$ be majority games with $H_{1}(G)=H_{1}\left(G^{\prime}\right)=H_{1}$ and $H_{2}(G)=$ $H_{2}\left(G^{\prime}\right)=H_{2}$. Naturally order the player $Q_{1}, \cdots, Q_{n}$ of the court $H_{1}$ and the players $R_{1}, \cdots, R_{n}$ of the court $H_{2}$. Since $H_{1}=H_{1}(G)$, there exist weights $w_{0} \geqq$ $\cdots \geqq w_{n}$ for $G$ and a one-to-one correspondence $\varphi$ mapping the last $n-1$ players $P_{2}, \cdots, P_{n}$, of $G$ upon $n-1$ of the players of $H_{1}$ so that the omitted player of $H_{1}$ is a member of the distinguished role and the numbers $w_{0}-w_{1}, w_{2}, \cdots, w_{n}$, assigned according to $\varphi$, are weights for $H_{1}$. By Lemma 1 and the definition of a role, there is a motion $\alpha$ of $H_{1}$ such that $\alpha\left(\varphi\left(P_{j}\right)\right)=Q_{j}$ for $j=2, \cdots, n$. By the same device we may regularize the correspondences of $G$ to $H_{2}, G^{\prime}$ to $H_{1}$ and $G^{\prime}$ to $H_{2}$, so that players with like indices from 2 to $n$ correspond. For any set $S$ of indices in $\{0,1, \cdots, n\}$, by considering the four possible values of $S \cap\{0,1\}$, one can give necessary and sufficient conditions for $\left\{P_{i} \mid i \in S\right\}$ to be a winning set in $G$, in terms of $H_{1}, H_{2}$, and $S$. The same conditions are necessary and sufficient for $\left\{P_{i}{ }^{\prime} \mid i \in S\right\}$ to win in $G^{\prime}$. Therefore the correspondence $P_{i} \leftrightarrow P_{i}{ }^{\prime}$ is an isomorphism between $G$ and $G^{\prime}$.

We call an $n$-player court $H_{1}$ and $n$-player majority game $H_{2}$ compatible if there exists a majority game $G$ such that $H_{1}(G)=H_{1}$ and $H_{2}(G)=H_{2}$. Evidently one can define a combinatorial scheme on the lines of a game, which must be the game $G$ if $G$ exists. The necessary and sufficient condition for compatibility is then the solvability of the system of linear inequalities defining weights for $G$, together with the axioms for a game. Reformulating, one has

THEOREM 1. An n-player court $H_{1}$ with naturally ordered players $Q_{i}$ and an n-player majority game $\mathrm{H}_{2}$ with naturally ordered players $R_{i}$ are compatible if and only if both

(a) whenever $\left\{Q_{i} \mid i \in S\right\}$ is a winning set in $H_{1}$ containing the player $Q_{1}$, then $\left\{R_{i} \mid i \in S\right.$ and $\left.i \neq 2\right\}$ is a winning set in $\mathrm{H}_{2}$, and

(b) there exist weights $u_{i}$ for $H_{1}, v_{i}$ for $H_{2}$, such that $u_{i}=v_{i}$ for $i=2, \cdots, n$. 
Proof. The necessity of (a) and (b) is obvious. Conversely; suppose (a) and (b) are valid. We may replace each $u_{i}$ with $\max \left(u_{i}, 0\right)$, and $v_{j}$ with $\max \left(v_{j}, 0\right)$, without affecting either game (since players with negative weight are dummies) or the validity of (a) and (b). We claim the numbers $\left(v_{1}+u_{1}\right) / 2,\left(v_{1}-u_{1}\right) / 2$, $u_{2}, \cdots, u_{n}$ are weights for a game $G$ with players $P_{0}, P_{1}, \cdots, P_{n}$. Call the subsets of $N=\left\{P_{0}, \cdots, P_{n}\right\}$ having more than half the total weight winning sets. For any $S \subset N$, not both $S$ and $N-S$ are winning. One must win unless they have the same weight; but in that case it is clear that two complementary sets in $H_{1}$ or in $\mathrm{H}_{2}$ would have the same weight, which is impossible. To prove that any superset of a winning set wins, it suffices to adjoin players one at a time. Adjoining a player other than $P_{1}$ cannot decrease the weight. Next suppose $S$ is a winning set containing $P_{0}$ and not $P_{1}$. Then the set $\left\{Q_{j} \mid j=1\right.$ or $j \geqq 2$ and $\left.P_{j} \epsilon S\right\}$ is a winning set in $H_{1}$, by computation; the set $\left\{R_{j} \mid j=1\right.$ or $j \geqq 2$ and $\left.P_{j} \in S\right\}$ is a winning set in $H_{2}$, by (a) and the fact that supersets of winning sets win in $H_{2}$; and $S \cup\left\{P_{1}\right\}$ wins in $G$, by computation. If $S$ is a winning set containing neither $P_{0}$ nor $P_{1}$, then $N-S$ contains both but fails to win; hence $N-S-\left\{P_{1}\right\}$ cannot win either, and $S \cup\left\{P_{1}\right\}$ is a winning set. Thus $G$ is a game, and therefore a majority game. To show that $H_{1}=H_{1}(G)$ and $H_{2}=H_{2}(G)$ it remains to prove that the ordering $\left(P_{\mathrm{c}}, \cdots, P_{n}\right)$ is natural. Replacing $P_{1}$ with $P_{0}$ never turns a winning set into a losing set, since $u_{1} \geqq 0$. If $S$ is a winning set containing $P_{2}$ and not $P_{1}$, and furthermore $S$ contains

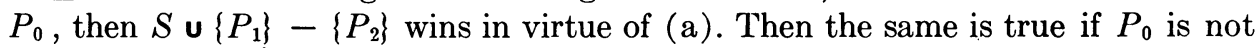
in $S$, by passage to the complement. For $i=2, \cdots, n-1$, the natural ordering of the players of $H_{1}$ implies that replacing $P_{i+1}$ by $P_{i}$ never turns a winning set into a losing set. Therefore we may rearrange the given weights for $G$ into weights $w_{0} \geqq \cdots \geqq w_{n}$ by successive transpositions, and the proof is complete.

The author's experience suggests that (b) of Theorem 1 may as well be ignored in computation; this is discussed in Section 4 below.

Corollary 1. For every $n$-player court $H_{1}$ there exists an $n$-player majority game compatible with $H_{1}$.

In fact there exists an $n$-player majority game compatible with all $n$-player courts. One system of weights for it is $(1,0, \cdots, 0)$; but as we noted before, any weights may be assigned to the $n-1$ dummies provided the first weight is large enough.

CoRollary 2. Let $f(n)$ be the number of different $n$-player majority games, $g(n)$ the number of these which have no dummies. Then $f(n+1) \geqq 2 f(n)-1$ and $g(n+1)$ $\geqq 2 g(n)-1$. For $n>6, f(n)>2^{n}$, and for $n>7, g(n)>2^{n}$.

Proof. Every majority game has at least two roles, with the exception of the games having weights $(1,1, \cdots, 1)$, of which there is one for each odd number of players. In any case the number of $n$-player courts is at least $2 f(n)-1$ and the number without dummies is at least $2 g(n)-1$. Corollary 1 and Lemma 2 show that there are at least as many corresponding $n+1$-player majority games; and clearly $G$ cannot have any dummies when $H_{1}(G)$ has none. Then if $f(n)>2^{n}$, it follows that $f(n+1)>2^{n+1}$; and the list at the end of the paper shows that $f(7)=135>2^{7}$. The same induction applies for $g$; and though $g(7)$ is only 114, the list shows there are over 256 corresponding courts.

The true order of $f$ and $g$ is not known. No respectable upper bound is known, 
though one can make slight improvements on $2^{2^{n}}$, which is the number of sets of sets of players. (Of course, the lower bound $2^{n}$ may be as wide of the mark.)

3. Efficient sequences. In this section we shall consider only games without dummies; it is also convenient to reverse our convention as to ordering. Let a sequence be a finite non-decreasing sequence of positive integers. For any sequence $w=$ $\left(w_{1}, \cdots, w_{n}\right)$, let $w(N)$ be the total $\Sigma_{i=1}^{i=n} w_{i}$; for any subset $S$ of $N=\{1, \cdots, n\}$, let $w(S)$ be $\Sigma_{i} \epsilon_{s} w_{i}$. Then $w(S)$ is called the weight of $S$.

Weights $\left(w_{i}\right)$ for a majority game are efficient provided $w(N)$ is an odd number $2 p-1$ and every subset of weight greater than $p$ contains a subset of weight exactly $p$. These conditions automatically make $\left(w_{i}\right)$ weights for a game, but the absence of zeros does not imply the absence of dummies. (Example: 1, 2, 2, 2.) We are assuming there are no dummies; that is, every index is in some set of weight $p$. Then it is known $[2,3]$ that the efficient weights are uniquely determined by the game, namely as minimum integral weights, and that their growth is restricted by the inequality

$$
w_{i} \leqq 1+\Sigma_{j=1}^{i-2} w_{j} .
$$

For any sequence $w$, let us call the non-negative integer $p$ acceptable for $w$ provided every set of weight $>p$ contains a subset of weight exactly $p$. Clearly 0 is acceptable for each $w$, no integer from 1 to $w_{n}-1$ can be acceptable, a sum of acceptable numbers is acceptable, and all sufficiently large numbers are acceptable.

LemMA 3. $p>0$ is acceptable for $\left(w_{1}, \cdots, w_{n+1}\right)$ if and only if both $p$ and $p-w_{n+1}$ are acceptable for $\left(w_{1}, \cdots, w_{n}\right)$.

Proof. If $p$ is acceptable for the long sequence it is clearly acceptable for a part of the sequence. If $p-w_{n+1}$ is not acceptable for $\left(w_{1}, \cdots, w_{n}\right)$, one has a set $S$ such that $w(S)>p-w_{n+1}$ and $S$ contains no subset of weight exactly $p-w_{n+1}$. Since the $w_{i}$ are increasing, one can assure that $w(S)<p$. Then $S u\{n+1\}$ can contain no subset of weight $p$, a contradiction. The converse is obvious.

Now let us call $p$ admissible for $w$ if both $p$ and $w(N)+1-p$ are acceptable; $p$ is proper if $1 \leqq p \leqq w(N)$. Let the sequence $w$ be efficient provided it satisfies (*) and some proper $p$ is admissible for $w$.

Theorem 2. A sequence $w$ is an efficient sequence if and only if $w$ is an initial segment of the (increasing) weights for some efficient weighted majority game.

Proof. If $w$ is an efficient sequence with proper admissible

$$
p \leqq p^{\prime}=w(N)+1-p,
$$

then $p+p^{\prime}$ is acceptable, hence admissible, for $\left(w_{1}, \cdots, w_{n}, p, p^{\prime}\right)$, by four applications of the lemma. To show that every index is in a set of weight $p+p^{\prime}$ it suffices, by efficiency, to show that this is true of the least index. Suppose $i$ is the least index actually used; if $i>1$ then by $(*), w_{i}$ is $\leqq$ the sum of the preceding weights, and $i$ can be replaced by a set of smaller indices without diminishing the weight, which reduces to a contradiction.

For the converse, $(*)$ is established in a previous paper [3], and the rest is clear from the lemma. Note that $(*)$ assures that for $n>1$, one of $p$ and $w(N)+1-p$ is $>w_{n}$, so that subtracting $w_{n}$ leaves a proper integer. 
TheOREM 3. Let $\left(w_{1}, \cdots, w_{n}\right)$ be efficient. Then $\left(w_{1}, \cdots, w_{n}, w_{n+1}\right)$ is efficient is and only if (1) $w_{n+1} \geqq w_{n},(2) w_{n+1} \leqq 1+\sum_{i=1}^{n-1} w_{i}$, and (3) $w_{n+1}=h-k$ for some $h$ and $k$ which are admissible for $\left(w_{1}, \cdots, w_{n}\right)$. A proper $p$ is admissible for

$$
\left(w_{1}, \cdots, w_{n+1}\right)
$$

if and only if both $p$ and $p-w_{n+1}$ are admissible for $\left(w_{1}, \cdots w_{n}\right)$.

Proof. The last statement follows from the lemma and the rest follows from that.

Note that since 0 is always admissible, the possible values of $w_{n+1}$ always include the proper admissible numbers. There are not always at least two of these. However, it is known [3] that there are at least $2^{n-4} n$-player games with efficient weights, for $n \geqq 4$. From Theorem 3 and the proof of Theorem 2 we see that the number of efficient sequences of length $n$ is an increasing function of $n$ and lies between the numbers of efficient game weights of length $n$ and of length $n+2$. In the other direction we have

Theorem 4. For an efficient sequence $\left(w_{1}, \cdots, w_{n}\right)$ there are at most $n$ values of $w_{n+1}$ which make $\left(w_{1}, \cdots w_{n+1}\right)$ efficient. Hence the number of efficient sequences of length $n$ is between $2^{n-4}$ and $(n-1)$ !.

Proof. There are at most $n$ acceptable integers $s_{k}$ between $w_{n}$ and $w(N)+1-w_{n}$, namely, $w_{n}, w_{n}+w_{n-1}, \cdots, w(N)$; for clearly if this sequence skips over $p$, $s_{k}<p<s_{k+1}$, then at step $k+1$ we have a set of weight $>p$ which drops below $p$ if its smallest element is removed. Now $w_{n+1}$ itself need not be acceptable, but $w(N)+1-w_{n+1}$ is. For there are $p$ and $p^{\prime}$ acceptable for $\left(w_{1}, \cdots, w_{n}\right)$ such that $p+p^{\prime}=w(N)+w_{n+1}+1$. By the lemma, both $p-w_{n+1}$ and $p^{\prime}-w_{n+1}$ are acceptable for $\left(w_{1}, \cdots, w_{\mathrm{p}}\right)$; hence so is their sum, which is $w(N)+1-w_{n+1}$. This establishes the inductive step, and a glance at the case $n=1$ establishes the upper bound $(n-1)$ !. The lower bound $2^{n-4}$ is established in [3] for $n \geqq 4$, and the cases $n=1,2,3$ are easily verified.

4. The enumeration. A method for generating all majority games of $n+1$ players from a list of all $n$-player majority games is as follows. List all the courst $(H, r)$, $H$ an $n$-player majority game and $r$ a role of $H$. For each $(H, r)$ there is at least one compatible game $\mathrm{H}_{2}$, as pointed out in Corollary 1 to Theorem 1 . All compatible games must be determined. In some cases (e.g. when $H_{2}$ has weights $(1,0, \cdots, 0)$ ) one can write down weights for the corresponding $n+1$-player game $G$ by rote. In general, however, one must screen the possible values of $\mathrm{H}_{2}$ and solve the systems of linear inequalities defining weights for $G$. For hand computation it seems best to apply the necessary condition (a) of Theorem 1, and for each $H_{2}$ satisfying that condition, to attempt to solve the inequalities for weights for $G$ with the side conditiont $w_{i} \geqq 0$. In a run such as the enumeration of the 7 -player majority games one develops subroutines; for example, there are different courts $H_{1}, H_{1}{ }^{\prime}$, such that $H_{2}$ is compatible with $H_{1}$ if and only if $H_{2}$ is compatible with $H_{1}^{\prime}$, and one may make mental lists of the corresponding classes of games $\mathrm{H}_{2}$.

It appears to be feasible though far from routine to code this method for automatic computation. No attempt has been made. For automatic computation it might be preferable simply to ignore the combinatorial conditions (a) and deal entirely in inequalities. However, (a) is a highly effective screen; for $n \leqq 6$ every pair $\left(H_{1}, H_{2}\right)$ satisfying (a) is compatible. 
The method expounded by von Neumann and Morgenstern [1] for enumerating all strong simple games involves, necessarily, a listing of winning sets (no other way is known for describing these games in general) ; and it also involves obtaining and then eliminating multiple isomorphic copies of each game. No other method is known for this problem. However, for generating a list of majority games, the present method is far superior. Note that if one were to employ the method of von Neumann and Morgenstern for this special purpose it would still be necessary to examine the inequalities determining whether each game has weights.

The recursive enumeration of all efficient sequences involves additional information which is most easily generated from the beginning, the efficient sequence $\left(w_{1}\right)$, where $w_{1}=1$. One may lay out three columns on a sheet of paper, the first giving the sequence $\left(w_{1}, \cdots, w_{n}\right)$, the second the list of all integers from 0 to $w(N)$ which are admissible for $w$, and the third the list of all differences of integers in the second column which satisfy the inequalities (1) and (2) of Theorem 3 . The numbers in the third column are precisely the values of $w_{n+1}$ which can be used for an extension $\left(w_{1}, \cdots, w_{n}, w_{n+1}\right)$; Theorem 3 tells how to compute the second column for $\left(w_{1}, \cdots, w_{n+1}\right)$, using the entries in the first column of this row and in the second column of the earlier row; then the new third column is computed from the new first and second columns. Coding this computation should be routine; the only complication is the irregularity of the size of the clumps of numbers.

It would certainly be desirable to develop a method for the enumeration of all majority games which, like the method for efficient sequences, should deal directly with arithmetic properties of the weights. A prerequisite would seem to be a scheme for assigning unique weights (not necessarily integers) to each game. The obvious idea of taking minimal integral weights is not enough, because these are not unique. To see this, consider the two sets of weights $(1,3,5,6,8,11,12,23,28,31,31,38)$ and $(1,3,5,7,8,11,12,23,28,31,31,37)$. One may verify that these weights determine the same game. One then proves twelve lemmas which show that any weights for this game are at least as large as $(1,3,5,6,8,11,12,23,28,31,31$, 37). First lemma: the first player is not a dummy; hence his weight in integers must be at least 1 . The proof that the second weight must be at least 3 involves finding combinations of $1,3,11$, and 12. Having all this, one observes that the twelve minimum values do not form weights for any game, since they permit a tie; there are no integers between them and the two sets of weights, and therefore both are minimal.

It can be shown by similar arguments that the 135 sets of weights listed below are all minimum. (Criteria from a previous paper [3] aid in most of the verifications.) In particular, no two of the 135 games are isomorphic. Comparable care has been taken to assure that no game is omitted. The list for $n \leqq 5$ is taken from von Neumann and Morgenstern [1], and the list for $n=6$ from Gurk and Isbell [2].

The 15 efficient weighted majority games of less than seven players

$\begin{array}{rrrr}1 & 11113 & 111114 & 111334 \\ 111 & 11122 & 111123 & 112225 \\ 1112 & 11223 & 111224 & 112335 \\ 1111 & 111112 & 111233 & \end{array}$

The 6 nonefficient weighted majority games of less than seven players

$\begin{array}{llll}111222 & 112234 & 122334 & 122345\end{array}$

112223 
The 23 seven-player efficient weighted majority games

$\begin{array}{rrrr}1111111 & 1111223 & 1112226 & 1122227 \\ 1111113 & 1111234 & 1112244 & 1122355 \\ 1111115 & 1111335 & 1112336 & 1122557 \\ 1111122 & 1111344 & 1112446 & 1123338 \\ 1111124 & 1111445 & 1113337 & 1123558 \\ 1111133 & 1112222 & 1113447 & \\ 1111223 & \text { The } 91 \text { seven-player nonefficient } & \text { weighted majority } & \text { games } \\ 1111333 & 1123345 & 1223344 & 1234557 \\ 1112224 & 1123356 & 1223346 & 1234568 \\ 1112233 & 1123446 & 1223348 & 1234579 \\ 1112235 & 1123457 & 1223357 & 1244567 \\ 1112334 & 1133344 & 1223445 & 1244679 \\ 1112345 & 1133355 & 1223447 & 2222333 \\ 1113335 & 1133445 & 1223449 & 2223334 \\ 1113346 & 1133456 & 1223456 & 2223345 \\ 1122223 & 1133467 & 1223458 & 2223367 \\ 1122225 & 1133557 & 1223467 & 2233344 \\ 1122234 & 1133568 & 1223559 & 2233445 \\ 1122236 & 1222233 & 1224457 & 2233456 \\ 1122245 & 1222235 & 1224558 & 2233478 \\ 1122333 & 1222334 & 1224569 & 2234455 \\ 1122335 & 1222336 & 1233345 & 2234556 \\ 1122337 & 1222345 & 1233446 & 2234567 \\ 1122344 & 1222347 & 1233455 & 2234589 \\ 1122346 & 1222356 & 1233457 & 2334456 \\ 1122445 & 1222455 & 1233468 & 2344567 \\ 1122447 & 1222556 & 1233556 & 2345678 \\ 1122456 & 1222567 & 1233567 & 3334455 \\ 1123334 & 1223335 & 1233578 & 3345567 \\ & 1223337 & 1234456 & \\ & & & \end{array}$

University of Washington, Seattle, Washington

1. J. Von Neumann \& O. Morgenstern, Theory of Games and Economic Behavior, Princeton Univ. Press, New Jersey, 1944.

2. H. M. GURK \& J. R. ISBELL, "Simple solutions," to appear in Annals of Mathematics Studies, Contributions to the Theory of Games, V. 4, Princeton Univ. Press, New Jersey.

3 . J. R. IsBeLL, "A class of majority games," Quart. Jn. of Math., Ser. 2, V. 7, 1956, p. 183-187. 